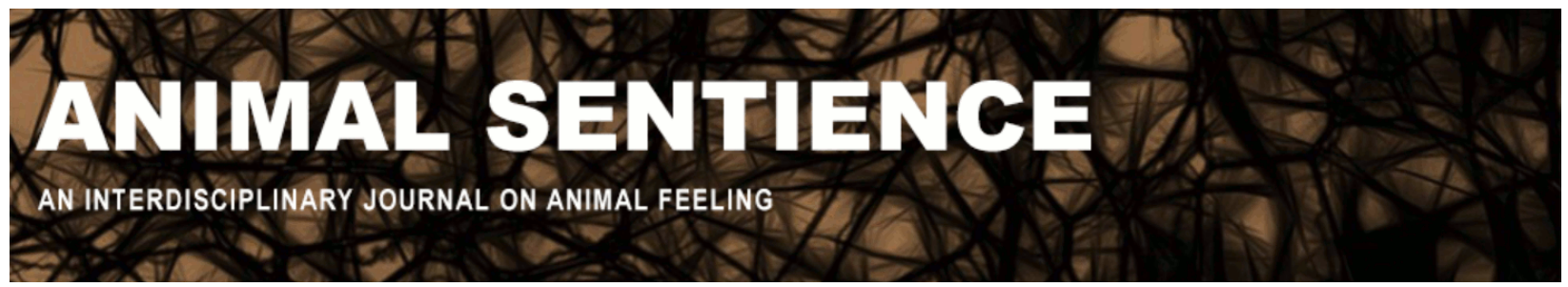

Corcoran, Andrew W. (2019) Cephalopod molluscs, causal models, and curious minds. Animal Sentience 26(13)

DOI: $10.51291 / 2377-7478.1493$

Date of submission: 2019-06-13

Date of acceptance: 2019-06-18 (c) 


\title{
Cephalopod molluscs, causal models, and curious minds
}

\author{
Commentary on Mather on Octopus Mind
}

\author{
Andrew W. Corcoran \\ School of Philosophical, Historical and International Studies \\ Monash University
}

\begin{abstract}
Mather (2019) presents a compelling case in favour of octopus mind. Surveying an impressive array of empirical literature, she identifies the creature's playful, inquisitive behaviour as emblematic of a distinctively mental form of agency. I offer an alternative perspective in which curiosity and play are construed as constitutive processes in the emergence of the (predictive) mind.
\end{abstract}

Andrew W. Corcoran, PhD candidate, Cognition and Philosophy Laboratory, Monash University, and academic research associate, Centre for Cognitive and Systems Neuroscience, University of South Australia, studies how biological systems adapt to various kinds of uncertainty. Website

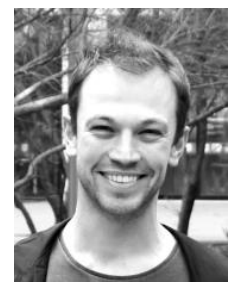

Mather (2019) argues that octopuses possess minds that orchestrate a suite of complex behaviours. A central plank of her argument rests on the assumption that intrinsic exploration and play are the products of a distinctively mental kind of intelligence. Granting that octopuses do indeed seek out novel, informative experiences for their own sake (but see Gutfreund, 2019), I propose an alternative view: Curiosity and play are not mere expressions of mind - they constitute its essential foundations.

I adopt a broad, but philosophically and neurobiologically informed, interpretation of mind as the product of an inference engine. On this view, minds are woven together from predictions generated by probabilistic models (Clark, 2016; Friston, 2010; Hohwy, 2013). Such models are tasked with inferring the (biologically relevant) causal relations that structure the organism's environment - both inside (i.e., internal milieu) and out (i.e., eco-niche or Umwelt).

A common objection to this thesis is that predictive minds ought to be averse to novelty or surprise. This follows from the idea that predictive minds seek to minimise the divergence between the sensory states they expect to encounter and those they actually experience. The thought goes that the easiest way to minimise this divergence ('prediction error') is to find a stable environment - the more devoid of stimulation the better - and to stay there, until death (Clark, 2018; Friston et al., 2012; Schwartenbeck et al., 2013).

One way of answering this argument is to point out that organisms' sensory environments include their internal (physiological) states, meaning that the reclusive creature is still subject to prediction errors generated from within (Corcoran \& Hohwy, 2018; Pezzulo et al., 2015). The octopus that sequesters itself deep inside a rocky crevice will accrue prediction error as its metabolic resources deplete, eventually compelling it to venture out in search of food. 
This line of reasoning does not account for the inquisitive behaviour described in the target article. Safe and sated octopuses seem intrinsically curious, motivated to explore their environments and interact with objects in ways that disclose their properties and affordances. Such information-seeking behaviour might seem puzzling from the perspective of minimising prediction error: Why should an organism designed to minimise prediction error pursue novel, surprising experiences in the absence of any obvious practical utility or extrinsic reward?

The apparent tension between information-seeking and surprise-avoiding can be resolved by considering the role of the generative model at the heart of the predictive mind. Such models evolved to infer the hidden (or latent) causes of the organism's sensory states as they unfold over time. Since most organisms inhabit complex, uncertain worlds, generative models often need to update their parameters in order to stay attuned to fluctuating environmental conditions. Novel experiences afford valuable opportunities to test how well model predictions 'generalise' to new sensory data, and to adjust the model when significant mismatches occur. Thus, while information-seeking behaviour might engender substantial amounts of prediction error in the short-term, such activity is vital for adapting the model in ways that improve the quality of its predictions (and thus minimise error) over longer timescales.

Calibrating model predictions against sensory evidence is a form of minimising prediction error that enables the agent to learn the biologically relevant causal dependencies structuring its environment. However, the capacity to adapt one's model in response to sensory input is not a distinctive property of mind per se. From a formal perspective, even a rock can be said to 'learn' about its environment in some minimal sense, insofar as it 'updates' or adapts its internal composition in response to external forces (e.g., ocean currents). Similarly, the environment itself can be described as a model that 'infers' or learns about the activities of its inhabitants over time (Bruineberg et al., 2018; Constant et al., 2018). Yet I do not wish to claim that rocks (or niches) harbour mental states.

These remarks suggest that minds are not characterised by the capacity to learn per se, but rather by the capacity to steer learning in a particular direction. Minded organisms are the architects of their own predictive models, actively sampling and interrogating their environments to procure informative sensory data (Gottlieb \& Oudeyer, 2018; cf. octopus head bobbing and tactile exploration; Mather, 2019). That is, minded agents autonomously solicit information that 'fills in the gaps' within their generative models (Friston et al., 2017; Schwartenbeck et al., 2019), thereby enhancing their epistemic grip on the world.

This analysis encourages one to view exploration and play not in terms of behavioural outputs that are 'controlled' or 'directed' by a mind (Mather, 2019), but rather as essential moments in the latter's realisation. Inquisitive and ludic forms of behaviour are ultimately in the service of discovery, revealing the causal contingencies that govern sensory experience (Buchsbaum et al., 2012; Gopnik, 2012), and disclosing novel possibilities for future action (Zylinski, 2015). Such intrinsically motivated 'epistemic actions' (Friston et al., 2015) might appear trivial or frivolous when taken in isolation, but function as essential mechanisms in transforming the subject of the generative model from a passive observer into an active mind.

There is a further, functional upshot of this analysis. The ability to seek out novel information about the latent processes structuring ecological dynamics (including how one's own actions impact on the causal order) - and thus to revise the generative model in ways that optimise its 'goodness of fit' with respect to these dynamics - should tend to engender 
predictions that enable the agent to adroitly navigate its niche over time. Equipped with a sufficiently accurate model of hidden states and contingent relations, the organism may exploit such knowledge to find creative solutions to novel, biologically salient problems that it encounters in the future. The inherent curiosity and playfulness of the predictive mind thus turns out to be functional (in an indirect, open-ended way) to the agent's long-term viability (Bateson, 2015), affording a 'general-purpose fuel for success' (Godfrey-Smith, 1996) that closes the loop between epistemic and pragmatic action.

Acknowledgments: AWC is supported by an Australian Government Research Training Program (RTP) scholarship and funding from the Centre for Cognitive and Systems Neuroscience, University of South Australia. The author wishes to thank Jakob Hohwy for insightful comments on a previous version of this manuscript.

\section{References}

Bateson, P. (2015). Playfulness and creativity. Current Biology, 25(1), R12-R16.

Bruineberg, J., Rietveld, E., Parr, T., van Maanen, L., \& Friston, K. J. (2018). Free-energy minimization in joint agent-environment systems: A niche construction perspective. Journal of Theoretical Biology, 455, 161-178.

Buchsbaum, D., Bridgers, S., Weisberg, D. S., \& Gopnik, A. (2012). The power of possibility: Causal learning, counterfactual reasoning, and pretend play. Philosophical Transactions of the Royal Society B, 367, 2202-2212.

Clark, A. (2016). Surfing uncertainty. Oxford: Oxford University Press.

Clark, A. (2018). A nice surprise? Predictive processing and the active pursuit of novelty. Phenomenology and the Cognitive Sciences, 17(3), 521-534.

Constant, A., Ramstead, M. J. D., Veissiere, S. P. L., Campbell, J. O., \& Friston, K. J. (2018). variational approach to niche construction. Journal of The Royal Society Interface, 15, 20170685.

Corcoran, A. W., \& Hohwy, J. (2018). Allostasis, interoception, and the free energy principle: Feeling our way forward. In M. Tsakiris \& H. De Preester (Eds.), The interoceptive mind: From homeostasis to awareness (pp. 272-292). Oxford: Oxford University Press.

Friston, K. J. (2010). The free-energy principle: A unified brain theory? Nature Reviews Neuroscience, 11, 127-138.

Friston, K. J., Lin, M., Frith, C. D., Pezzulo, G., Hobson, J. A., \& Ondobaka, S. (2017). Active inference, curiosity and insight. Neural Computation, 29, 2633-2683.

Friston, K. J., Rigoli, F., Ognibene, D., Mathys, C., FitzGerald, T. H. B., \& Pezzulo, G. (2015). Active inference and epistemic value. Cognitive Neuroscience, 6(4), 187-214.

Friston, K. J., Thornton, C., \& Clark, A. (2012). Free-energy minimization and the dark-room problem. Frontiers in Psychology, 3, 130.

Godfrey-Smith, P. (1996). Complexity and the function of mind in nature. Cambridge: Cambridge University Press.

Gopnik, A. (2012). Scientific thinking in young children: Theoretical advances, empirical research, and policy implications. Science, 337, 1623-1627.

Gottlieb, J., \& Oudeyer, P-Y. (2018). Towards a neuroscience of active sampling and curiosity. Nature Reviews Neuroscience, 19, 758-770. 
Gutfreund, Y. (2019). Who needs a mind when you have thousands of fingers? Animal Sentience 26(3).

Hohwy, J. (2013). The predictive mind. Oxford: Oxford University Press.

Mather, J. (2019). What is in an octopus's mind? Animal Sentience 26(1).

Pezzulo, G., Rigoli, F., \& Friston, K. J. (2015). Active inference, homeostatic regulation and adaptive behavioural control. Progress in Neurobiology, 134, 17-35.

Schwartenbeck, P., FitzGerald, T. H. B., Dolan, R. J., \& Friston, K. J. (2013). Exploration, novelty, surprise, and free energy minimization. Frontiers in Psychology, 4, 710.

Schwartenbeck, P., Passecker, J., Hauser, T. U., FitzGerald, T. H. B., Kronbichler, M., \& Friston, K. J. (2019). Computational mechanisms of curiosity and goal-directed exploration. eLIFE, 8, e41703.

Zylinski, S. (2015). Fun and play in invertebrates. Current Biology, 25(1), R10-R12. 